\title{
AMENDMENTS
}

\section{Author Correction: A lipid-based partitioning mechanism for selective incorporation of proteins into membranes of HIV particles}

Prabuddha Sengupta (D), Arnold Y. Seo, H. Amalia Pasolli, Yul Eum Song, Marc C. Johnson and

Jennifer Lippincott-Schwartz $\mathbb{D}$

Correction to: https://doi.org/10.1038/s41556-019-0300-y, published online 1 April 2019

In the version of this article originally published, the name of co-author Marc C. Johnson was missing the middle initial. The middle initial 'C.' has been added in the author list as well as in the 'author contributions' section (as M.C.J.). The error has been corrected in the PDF and HTML versions of the paper.

Published online: 10 April 2019

https://doi.org/10.1038/s41556-019-0327-0

\section{Author Correction: Sin1 phosphorylation impairs mTORC2 complex integrity and inhibits downstream Akt signalling to suppress tumorigenesis}

Pengda Liu, Wenjian Gan, Hiroyuki Inuzuka, Adam S. Lazorchak, Daming Gao, Omotooke Arojo, Dou Liu, Lixin Wan, Bo Zhai, Yonghao Yu, Min Yuan, Byeong Mo Kim, Shavali Shaik, Suchithra Menon, Steven P. Gygi, Tae Ho Lee, John M. Asara, Brendan D. Manning, John Blenis, Bing Su and Wenyi Wei

Correction to: Nature Cell Biology https://doi.org/10.1038/ncb2860, published online 27 October 2013.

In the version of this Article originally published, the labels for Rictor and mTOR in the whole cell lysate (WCL) blots were swapped in Fig. $3 \mathrm{~b}$ and the mTOR blot was placed upside down. Unprocessed blots of mTOR were also missing from Supplementary Fig. 9. The corrected Figs are shown below. In addition, control blots for the mTOR antibody (Cell Signalling Technology \#2972) were also missing. These are now provided below, as Fig. 9, and show that the lower band is likely non-specific.
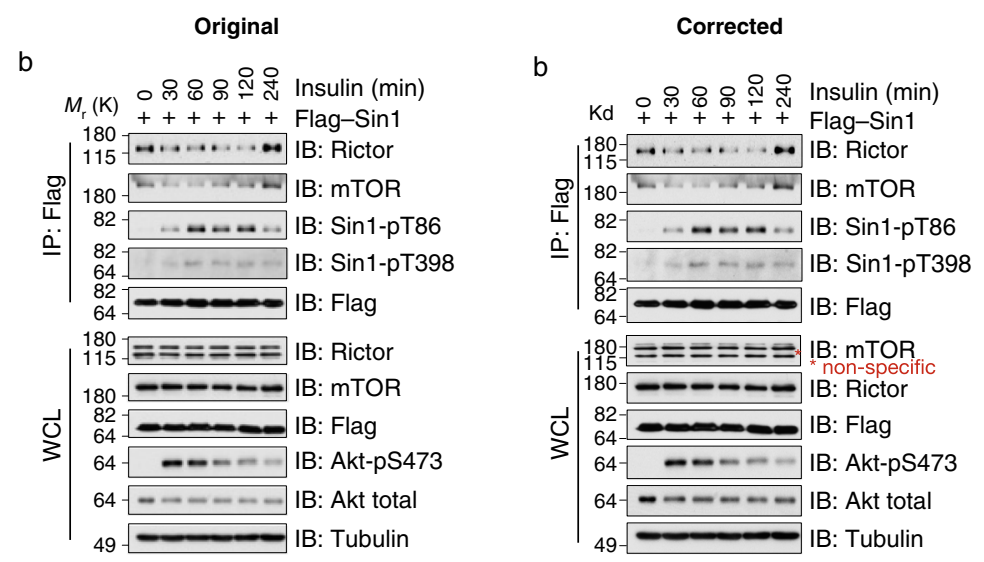

Fig. 3 | Original and Corrected. 